DRAFT VERSION OCTOBER 24, 2018

Preprint typeset using LTEX style emulateapj v. 08/22/09

\title{
RELATIVE ROLE OF STARS AND QUASARS IN COSMIC REIONIZATION
}

\author{
Marta Volonteri ${ }^{1}$ \& Nickolay Y. Gnedin ${ }^{2,3,4}$ \\ Draft version October 24, 2018
}

\begin{abstract}
We revisit the classical view that quasar contribution to the reionization of hydrogen is unimportant. When secondary ionization are taken into account, in many plausible scenarios for the formation and growth of supermassive black holes quasars contribute substantially or even dominantly at $z \gtrsim 8$, although their contribution generally falls below that of star-forming galaxies by $z=6$. Theoretical models that guide the design of the first generation of redshifted $21 \mathrm{~cm}$ experiments must, therefore, substantially account for the quasar contribution in order to be even qualitatively accurate.
\end{abstract}

Subject headings: cosmology: theory - cosmology: large-scale structure of universe - galaxies: formation galaxies: intergalactic medium

\section{INTRODUCTION}

Modeling theoretically the properties of the sources that reheated and re-ionized the intergalactic medium at the end of the cosmic Dark Ages is difficult, because, at present, our knowledge of the nature of ionizing sources is highly incomplete. The conventional view, held over a decade since the original work of Shapiro \& Giroux (1987); Shapiro et al. (1994); Giroux \& Shapiro (1996a); Madau et al. (1999), is that the ionization at the highest redshifts is dominated by the UV radiation from star-forming galaxies, and that the non-thermal contribution from quasars builds up only later, at $z<4$, to dominate the double ionization of helium (which requires photons of energies exceeding $54.4 \mathrm{eV}$ ).

This conventional view, however, is incomplete. In particular, it ignores an important physical process of "secondary ionizations" by energetic ionizing photons. It is a well-known physical fact that an ionizing photon with the energy $E$ in excess of $\sim 100 \mathrm{eV}$ is capable of ionizing more than a single atom - the excess photon energy of $E-13.6 \mathrm{eV}$ is deposited in the electron, which is capable of ionizing one or more additional atoms in its vicinity (Shull \& van Steenberg 1985; Valdés \& Ferrara 2008).

These secondary ionizations can substantially increase the relative contribution of quasars to the reionization of the universe. For example, a $1 \mathrm{keV}$ X-ray photon can ionize up to $\sim 25$ hydrogen atoms. While this important effect has been included in many of the prior work on hydrogen reionization (Giroux \& Shapiro 1996b; Oh 2001; Venkatesan et al. 2001; Ricotti et al. 2002; Machacek et al. 2003; Ricotti \& Ostriker 2004; Dijkstra et al. 2004a; Madau et al. 2004; Furlanetto 2006; Zaroubi et al. 2007; Pelupessy et al. 2007; Salvaterra et al. 2007; Cohn \& Chang 2007; Thomas \& Zaroubi 2008; Kramer \& Haiman 2008; Shull \& Venkatesan 2008; Warszawski et al. 2008; Schleicher et al. 2008; Santos et al. 2008; Ripamonti et al. 2008), it may be worth re-assessing the canonical view of

\footnotetext{
${ }^{1}$ Department of Astronomy, University of Michigan, 500 Church Street, Ann Arbor, MI, USA; martav@umich.edu

2 Particle Astrophysics Center, Fermi National Accelerator Laboratory, Batavia, IL 60510, USA; gnedin@fnal.gov

${ }^{3}$ Kavli Institute for Cosmological Physics, The University of Chicago, Chicago, IL 60637, USA

${ }^{4}$ Department of Astronomy \& Astrophysics, The University of Chicago, Chicago, IL 60637 USA
}

the sub-dominance of the quasar population as the source of hydrogen reionization in light of recent improvements in the values of cosmological parameters and new developments in our understanding of the growth of supermassive black holes at high redshifts.

There are two possible approaches to modeling reionization. In the first one, a model for the emission, propagation, and absorption of ionizing radiation from various categories of sources is constructed. Ultimately, such a model must involve a cosmological numerical simulation that resolves Lyman limit systems with sub-kpc resolution and clustering of galaxies and quasars on $\sim 100 \mathrm{Mpc}$ scales. Such a large uniform dynamic range is not yet feasible in modern cosmological simulations, so this type of modeling inevitably suffers from the unknown systematic biases, but, at the same time, it offers the most complete model of cosmic reionization.

A second, much simpler (and, therefore, much more limited) approach consists in ignoring the absorption and propagation of ionizing photons, and restricting a theoretical model to only counting the number of ionizations per atom. A major (but not the sole) limitation of this approach is that the criterion for reionization - the required number of ionizations per atom - is unknown. While reasonable estimates for this number can be constructed (e.g. Gnedin 2008, and discussion there), it, at the very least, must exceed unity ${ }^{5}$.

In this brief paper, we adopt the second, simple approach, to couple theoretical expectations for both stellar sources and quasars, and to consider their relative contribution to the total number of ionizations per hydrogen atom. In line with our limited goals, we restrict our attention only to two main astrophysical types of ionizing sources, although we remain acutely aware that other, more exotic sources - including the energetic photons and electrons from dark matter annihilation - can provide a substantial or even dominant contribution to the total ionization budget (e.g. Belikov \& Hooper 2009, and references therein).

\section{METHOD}

We evolve the population of two distinct types of seed massive black holes (MBHs): either "small seeds", derived from Population III remnants (Madau \& Rees 2001;

\footnotetext{
5 Or, even more precisely, about 0.97 , as a few percent of gas remains neutral after reionization, locked in Lyman limit and Damped Ly $\alpha$ systems.
} 
Volonteri et al. 2003), or "large seeds", derived from gasdynamical collapse in metal-free galaxies (Begelman et al. 2006; Lodato \& Natarajan 2006). We adopt one single formation scenario for each realization of the Universe we consider, therefore each model contains only "small seeds" or "large seeds".

In the Population III remnants model, seed MBHs form with masses $m_{\text {seed }} \sim$ few $\times 10^{2} \mathrm{M}_{\odot}$ (see Fig. 1, top left panel), in haloes collapsing at $z>15$ from rare 3.5- $\sigma$ peaks of the primordial density field (Volonteri et al. 2003).

In the "large seeds" scenario, massive seeds with $M \approx$ $10^{4} \mathrm{M}_{\odot}$ can form at high redshift, when the intergalactic medium has not been significantly enriched by metals. Here we refer to Begelman et al. (2006); Lodato \& Natarajan (2006), for more details of the physical MBH formation model. Seeds form in gravitationally unstable pre-galactic disk with primordial composition, in halos with virial temperature $\sim 10^{4} \mathrm{~K}$, cooled mainly by atomic hydrogen. The stability of gaseous disks depends on two parameters, the halo spin parameter $\lambda_{\text {spin }}$, and the fraction of baryonic matter that ends up in the disk, $f_{d}=\left(\Omega_{M} / \Omega_{b}\right)\left(M_{\text {disk }} / M_{h}\right)$. A maximum spin parameter $\lambda_{\text {spin,max }}$ exists, for which a disk is unstable as a function of the fraction of baryons forming the disk, i.e., for every $f_{d}$, disks are stable for $\lambda_{\text {spin }}>\lambda_{\text {spin,max }}$. We here assume $\left(f_{d}, \lambda_{\text {spin,max }}\right)=(0.2,0.2)$. The mass of the forming MBH seeds is set by the joint characteristics of the gas flow and of the evolution of the collapsed gas (Begelman et al. 2007). The mass function of $\mathrm{MBH}$ seeds peaks at $10^{4} \mathrm{M}_{\odot}$ (see Fig. 1, top right panel).

We study $\mathrm{MBH}$ evolution within dark matter halos via a Monte-Carlo algorithm based on the extended PressSchechter formalism. The population of MBHs evolves along with their hosts according to a "merger driven scenario", as described in Volonteri et al. (2003; 2009). An accretion episode is assumed to occur as a consequence of every major merger (mass ratio larger than 1:10) event. During an accretion episode, each $\mathrm{MBH}$ accretes an amount of mass, $\Delta M=$ $9 \times 10^{7} \mathrm{M}_{\odot}(\sigma / 200 \mathrm{~km} / \mathrm{s})^{4}$, that scales with the $M_{\mathrm{BH}}-\sigma_{*}$ relation of its hosts (see Volonteri \& Natarajan 2009). Accretion starts after a dynamical timescale and lasts until the $\mathrm{MBH}$ has accreted $\Delta M$.

We model the accretion rate onto MBHs in two ways. As a baseline model, we assume that accretion proceeds at the Eddington rate (see also Salvaterra et al. 2007). In a second case, we model the accretion rate during the active phase from the extrapolation of the empirical distribution of Eddington ratios, $\lambda=\log \left(L_{\mathrm{bol}} / L_{\text {Edd }}\right)$, found in Merloni \& Heinz (2008). We adopt a fitting function of the Eddington ratio distribution as a function of MBH mass and redshift (Merloni 2009). We are here extrapolating such a model at much higher redshifts and lower MBH masses than originally intended. We caution readers in taking the results of this model face value.The main goal of our exercise is to probe possible sensible ranges for the accretion rates on $\mathrm{MBHs}$.

At each timestep of our Monte Carlo simulation of halos merger trees, we calculate the average energy density emitted during each timestep by the $\mathrm{MBH}$ population as follows:

$$
\Delta U=\langle\epsilon\rangle \Delta \rho_{a c c} c^{2}
$$

where $\Delta \rho_{a c c}$ is the total mass density (in comoving units) accreted by MBHs within the timestep, and $\langle\epsilon\rangle$ is the average radiative efficiency, which we assume depends solely on $\mathrm{MBH}$ spins for radiatively efficient accretors. We evolve $\mathrm{MBH}$

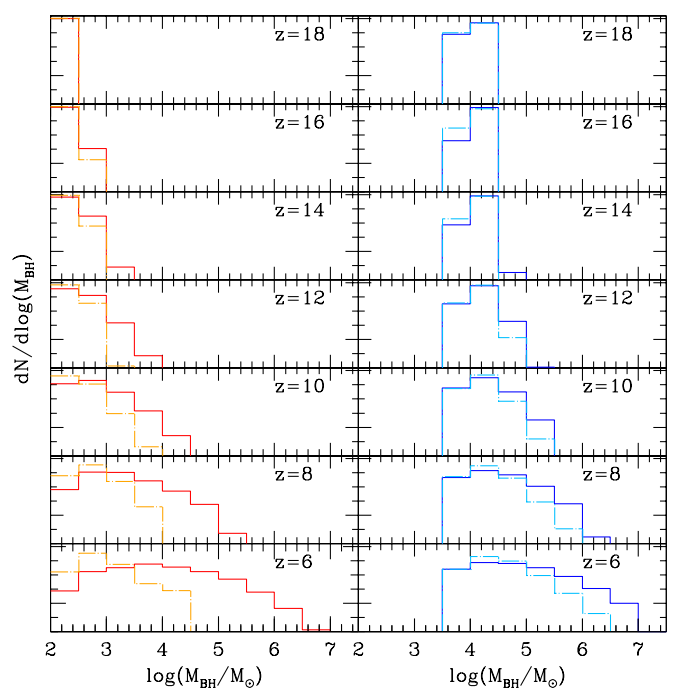

FIG. 1. - Mass function of MBHs at different cosmic times. Top to bottom: $18<z<20 ; 16<z<18 ; 14<z<16 ; 12<z<14 ; 10<z<12$; $8<z<10 ; 6<z<8$. Left panels: small seeds. Right panels: large seeds. Solid histogram: Eddington accretion; dot-dashed histogram: Merloni-Heinz accretion.

spins according to two simple models: coherent accretion (Volonteri et al. 2005) and chaotic accretion (King \& Pringle 2007). These two models lead to rapidly spinning MBHs ("high spins", $\langle\epsilon\rangle=0.2$ ), and slow rotators ("low spins", $\langle\epsilon\rangle=0.06)$ respectively. We assume that $\mathrm{MBH}$ are born nonspinning.

The energy density, $U$, depends on both the accretion rate (in Eddington units, $f_{E d d}=10^{\lambda}$ ) and $\epsilon$. Assuming constant $f_{E d d}$ and $\epsilon$, at a given time $t$ :

$$
U(t) \propto \epsilon M_{\text {seed }}\left(e^{f_{E d d} \frac{1-\epsilon}{\epsilon} \frac{t}{\tau}}-1\right)
$$

where $M_{\text {seed }}$ is the average mass of MBH seeds in each scenario, and $\tau=0.45 \mathrm{Gyr}$ is the Eddington timescale. We can see that if $t \ll \tau: U \propto f_{E d d}(1-\epsilon)$. Within our evolutionary scheme $\epsilon$ is determined for each MBH, while we have used a single, average, $\langle\epsilon\rangle$ in calculating $U$. Since it takes a few Myr for MBHs in the coherent accretion model to spin-up to large spins (i.e. for the radiative efficiency to increase from $0.06-$ Schwarzschild hole, to $0.2-$ spin $=0.9$ ) this scheme slightly overestimates the radiative output from MBHs in the 'high spin' case at $z>11$.

We further assume that a fixed fraction $f_{\mathrm{UV}}$ of the bolometric power radiated by high-redshift quasars is emitted as hydrogen-ionizing photons. The number of ionizing photons scales as $f_{\mathrm{UV}} / E_{\gamma}$, where $E_{\gamma}$ is the mean photon energy (see section 2). We refer the reader to Madau et al. (2004) for a thorough discussion about the quasar spectra; in the following we assume, conservatively, that $f_{\mathrm{UV}}=0.2$.

The total comoving energy density of ionizing radiation emitted by the growing MBH population is then:

$$
U_{\gamma}(z)=\sum_{i=0, j(z)} f_{\mathrm{UV}} \Delta U_{i}
$$

where the sum is over the timesteps between our starting redshift $\left(z_{\max }=20\right.$, timestep 0$)$, and the timestep, $j(z)$ corresponding to the redshift of interest, $z$.

We have also implemented a more pessimistic case for 
MBH accretion, loosely based on Milosavlievic et al. (2008), where the accretion rate has been fixed to $30 \%$ of the Eddington accretion rate. In this scenario, the yield in ionizing radiation is lower than for the case of $100 \%$ Eddington rate, but ionization histories in this scenario fall within the range spanned by our other models; they, thus, do not change any of our conclusions and we do not consider them further in this paper.

Our models are consistent with the constraints from the soft X-ray background (Dijkstra et al. 2004b). Our predicted population of high redshift AGN would account for almost $5 \%$ of the measured $(0.5-8 \mathrm{keV})$ background, or $\sim 25 \%$ of the unresolved one (Salvaterra et al. 2005, 2007). Models are also consistent with the bolometric luminosity function of quasars at $z>4$ (Hopkins et al. 2007). "Large seeds" are preferred as our fiducial model, based on a better agreement with the bolometric luminosity function at $z \sim 4$, however we note that in this paper we probe much higher redshifts than those probed by the study of Hopkins et al. (2007), hence we consider the match with the luminosity function at lower redshifts as a weak constraint.

For the stellar contribution to the total ionizing background we use the extrapolation of the observed UV luminosity functions of high redshifts galaxies (Bouwens et al. 2007, 2008) and the assumed value for the relative (to the amount of escaped UV light at 1000) escape fraction of ionizing radiation. The complete details of the methodology are described in Gnedin (2008). Here we only briefly repeat that the total mass-to-light ratios of higher redshift galaxies are computed in a given cosmology by matching the observed spatial abundance of galaxies of a given luminosity to the theoretically computed abundance of halos of a given mass, so that

$$
n\left(>L_{\mathrm{UV}}\right)=n\left(>M_{\mathrm{TOT}}\right)
$$

(and, optionally, an additional factor that accounts for "bursty" star formation rate in high redshift galaxies can be incorporated in eq. [4]; reasonable values for that factor make insignificant impact on our results).

The obtained thus mass-to-light ratio can be extrapolated to higher redshifts. Since the mass-to-light ratio is a weak function of redshift, the uncertainty of such an extrapolation does not dominate the final uncertainty of our estimate for the ionizing emissivity from galaxies. Instead, the final uncertainty is dominated by the uncertainty (both observational and theoretical) on the adopted value of the relative escape fraction for ionizing radiation (Gnedin 2008).

The combined (stellar plus quasar) contribution to reionization can then be estimated as

$$
N_{\gamma / a}=N_{\gamma / a, *}+\frac{U_{\gamma}}{E_{\gamma} n_{a}}+f_{\mathrm{SI}}(x) \frac{U_{\gamma}}{14.4 \mathrm{eV} n_{a}},
$$

where $N_{\gamma / a}$ is the number of ionizations per atom, $n_{a}=(1-$ $\left.0.75 Y_{p}\right) n_{b} \approx 2.0 \times 10^{-7} \mathrm{~cm}^{-3}$ is the comoving number density of atoms of hydrogen or helium (we assume that most of helium is only singly ionized during hydrogen ionization), and $14.4 \mathrm{eV}$ is the mean ionization energy per atom. The first term in this equation accounts for the contribution from stars, the second one includes primary ionizations by ionizing photons from quasars with the mean photon energy $E_{\gamma}$, and the last term accounts for secondary ionizations from energetic ionizing photons. For the gas uniformly ionized to the ionization fraction $x$, the fraction of radiation energy density $f_{\mathrm{SI}}$ going into secondary ionizations has been computed by
Shull \& van Steenberg (1985), and their results can be conveniently fitted by a simple but accurate formula (Ricotti et al. 2002)

$$
f_{\mathrm{SI}}(x) \approx 0.35\left(1-x^{0.4}\right)^{1.8}-1.77\left(\frac{28 \mathrm{eV}}{E_{\gamma}}\right)^{0.4} x^{0.2}\left(1-x^{0.4}\right)^{2}
$$

In reality, of course, the universe is not uniformly ionized, so the quantity $x$ in equation (5) is an effective value $x_{\text {eff }}$, such that

$$
N_{\gamma / a}\left(x_{\text {eff }}\right)=\left\langle N_{\gamma / a}(x)\right\rangle
$$

and the average is mass-weighted. Thus, $x_{\text {eff }}$ is not a mass- or volume-weighted average of the cosmic ionization fraction. However, $x_{\text {eff }}=0$ for the fully neutral and $x_{\text {eff }}=1$ for the fully ionized universe.

The quantity $N_{\gamma / a, *}$ has been computed in Gnedin (2008) and is not discussed here. The mean energy of ionizing photons from quasars, $E_{\gamma}$, remains a parameter of our model. The exact value of this parameter obviously depends on the spectral shape of the quasar energy distribution. If we assume a classic multicolor disk spectrum up to $k T_{\max } \sim$ $1 \mathrm{keV}\left(M_{\mathrm{BH}} / \mathrm{M}_{\odot}\right)^{-1 / 4}$ (Shakura \& Sunyaev 1973), and a nonthermal power-law component with spectral slope $L_{\nu} \propto \nu^{-\alpha}$, with $\alpha \approx 1$ at higher energies, we find $E_{\gamma} \simeq 300 \mathrm{eV}$ for $M_{\mathrm{BH}}=$ $10^{3} \mathrm{M}_{\odot}$. The spectrum is harder/softer for smaller/larger $\mathrm{MBH}$ masses. As a fiducial value, we adopt $E_{\gamma}=300 \mathrm{eV}$ (cfr. Fig 1), and we investigate the sensitivity of our results to the precise value of this parameter in the next section.

Equation (5) cannot be evaluated without an equation for $x_{\text {eff }}$ as a function of time. A complete computation of $x_{\text {eff }}(t)$ requires a sophisticated three-dimensional modeling of the transfer of ionizing radiation throughout the inhomogeneous gas density distribution in the universe. Since such modeling is well beyond the scope of this paper, we instead introduce the following ansatz for $x_{\text {eff }}(t)$ :

$$
x_{\text {eff }}=\min \left(1, f_{n \rightarrow x} N_{\gamma / a}\right),
$$

where $f_{n \rightarrow x}$ is a parameter. The motivation of this ansatz follows from the realization that if every atom in the universe is ionized exactly once, then in the beginning of reionization, while $x_{\text {eff }}$ is sufficiently small, $x_{\text {eff }} \approx N_{\gamma / a}{ }^{6}$ The factor $f_{n \rightarrow x}$ therefore accounts for the loss of ionizing photons to recombinations and for more complicated dependence of $x_{\text {eff }}$ on $\langle x\rangle$ at the later stages of reionization. This factor cannot be too small (or the universe would never be reionized), and any value for $f_{n \rightarrow x}$ above about 0.5 (half of photons lost for recombination) does not affect our conclusions. Therefore, in the rest of this paper we adopt $f_{n \rightarrow x}=0.75$ as our fiducial value. In reality, $f_{n \rightarrow x}$ must be a function of time, but since it is not likely to be much lower than 1 , the exact time dependence of $f_{n \rightarrow x}$ is unimportant at the level of precision of our approximations (which is dominated by the uncertainties on the escape fraction from galaxies and the lack of knowledge for the specific parameters of our quasar model).

\section{RESULTS}

In the rest of this paper we consider two different cosmological models. The first one (which we call "WMAP-5") is the most likely joint fit to the fifth year WMAP data, Baryonic

\footnotetext{
6 If we ignore the second term in equation 6 which is small for $E_{\gamma} \gg$ $100 \mathrm{eV}$, and expand $f_{\mathrm{SI}}$ in Taylor series around $x=0$, then for sufficiently small $x, x_{\text {eff }}=\langle x\rangle$.
} 
TABLE 1

Cosmological Parameters

\begin{tabular}{lcc}
\hline \hline Parameter & WMAP-5 & WMAP-5UP \\
\hline$\Omega_{m}$ & 0.28 & 0.28 \\
$h_{100}$ & 0.7 & 0.7 \\
$\sigma_{8}$ & 0.82 & 0.84 \\
$n_{S}$ & 0.96 & 0.98 \\
& & \\
\hline
\end{tabular}

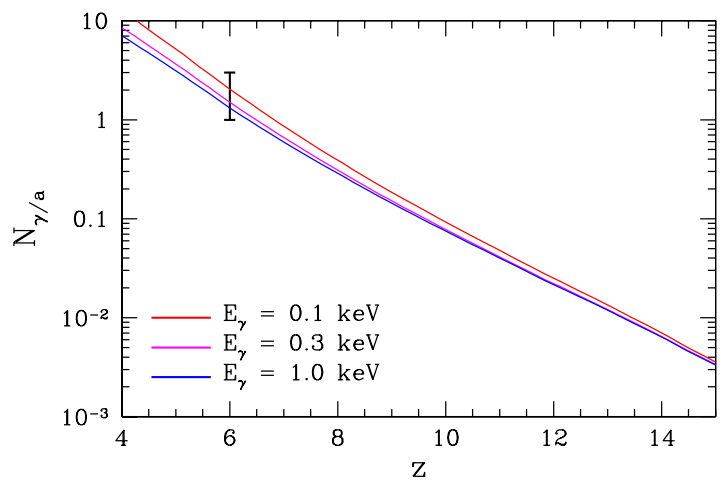

FIG. 2.- The total ionizations-to-atom ratio as a function of redshift for our fiducial model ("large seeds, high spins" in WMAP-5UP cosmology) for 3 values of the mean energy of ionizing photons from quasars $E_{\gamma}$. The adopted reionization criterion, $1<N_{\gamma / a}<3$ at $z=6$, is shown as a thick black segment with error-bars.

Acoustic Oscillations from SDSS, and supernovae, as presented in the second column of Table 1 from Komatsu et al. (2009). The second model ("WMAP-5UP") is obtained from the first one by increasing both the amplitude of the density fluctuations $\sigma_{8}$ and the scalar spectral index $n_{S}$ upward by 1 standard deviation. The relevant values of cosmological parameters for these two models are listed in Table 1

Figure 2 demonstrates the sensitivity of the total ionizations-to-atom ratio $N_{\gamma / a}$ on the assumed value for the mean energy of ionizing photons from quasars $E_{\gamma}$. Because ionizations from the quasar population are dominated by secondary ionizations (which are independent of $E_{\gamma}$ ) for any plausible value of $E_{\gamma}$, this parameter has only a mild effect on our results.

Figures 3 and 4 now present our main result: the computed $N_{\gamma / a}$ as a function of redshift for two cosmologies and various parameters of the quasar model. The criterion for the universe to be reionized at $z=6\left(1<N_{\gamma / a}<3\right)$ we adopt from Gnedin (2008), where it is discussed and justified.

Clearly, the WMAP5 model (with the most likely values of $\sigma_{8}$ and $n_{S}$ ) is somewhat short of the reionization requirement. As has been discussed by Gnedin (2008), this is not necessarily a serious problem, since the likely uncertainty on our extremely simple model is a factor of 2 or so. Never-the-less, slightly higher values for $\sigma_{8}$ and/or $n_{S}$ provide a wider breathing space for reionization modeling.

In order to illustrate a plausible estimate for the uncertainties due to observational errors on the escape fraction (c.f. Shapley et al. 2006), high redshift luminosity functions (Bouwens et al. 2007), etc, we show in Figure 5 the "large seeds - high spin" quasar model in the WMAP-5UP cosmology (which we adopt as our "fiducial model") together with the estimated errors. Notice, that formally Bouwens et al. (2007) galaxy luminosity function at $z>6$ should be con-
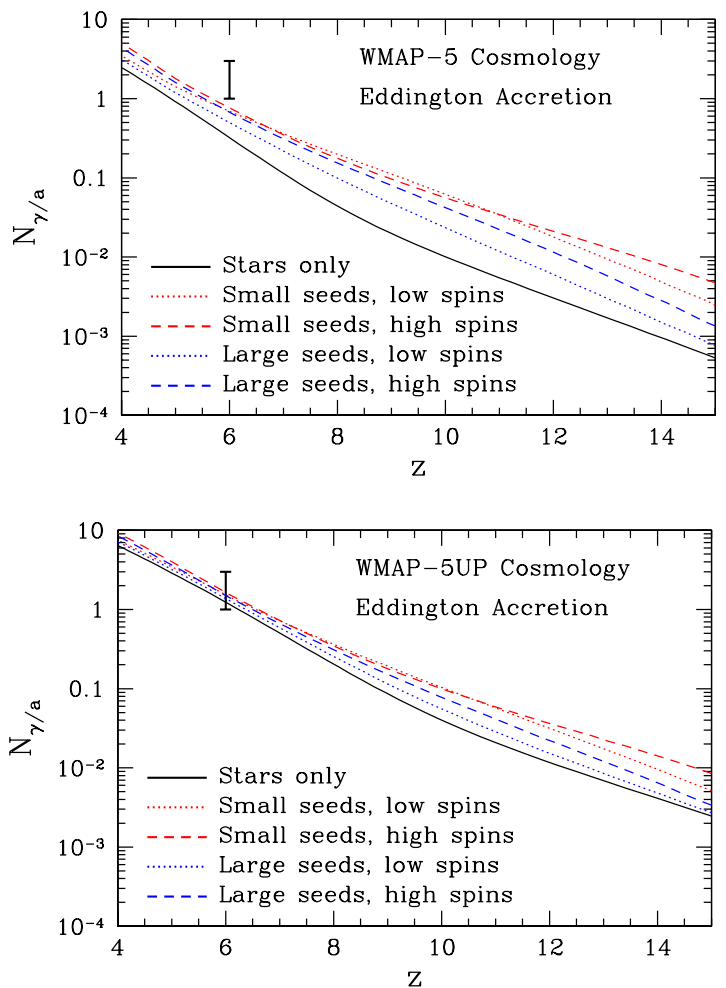

FIG. 3.- The total ionizations-to-atom ratio as a function of redshift for different models for quasar contribution (red and blue lines) as well as contribution from the stars alone (black solid line) for the Eddington accretion model for two chosen cosmologies. The adopted reionization criterion, $1<N_{\gamma / a}<3$ at $z=6$, is shown as a thick black segment with error-bars.
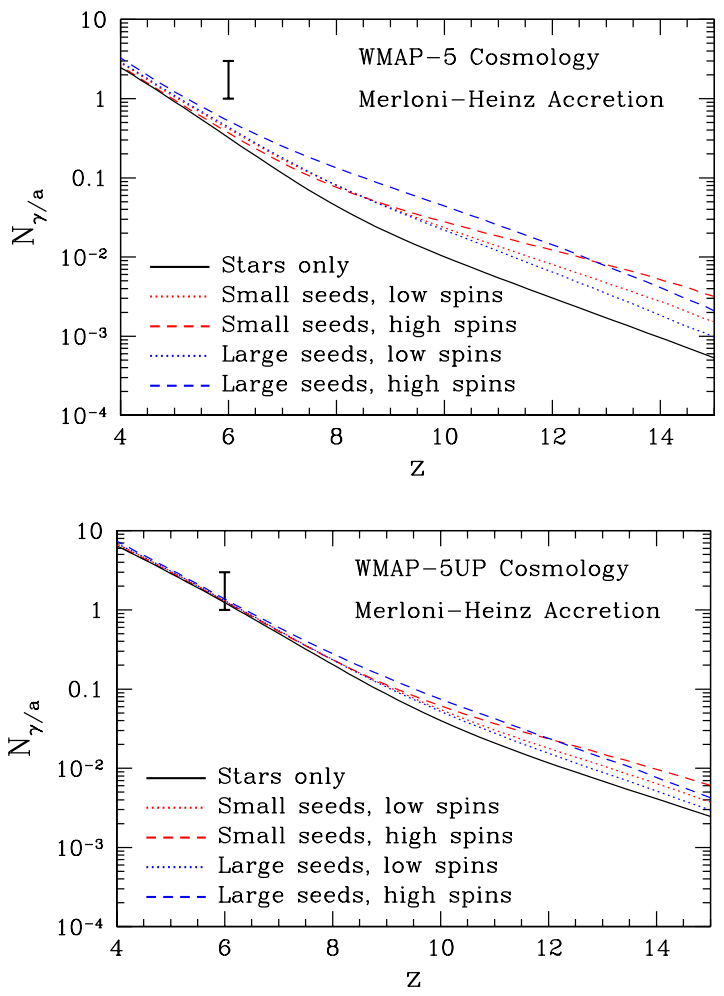

FIG. 4.- Same as Fig. 4 but for the Merloni \& Heinz accretion model. 


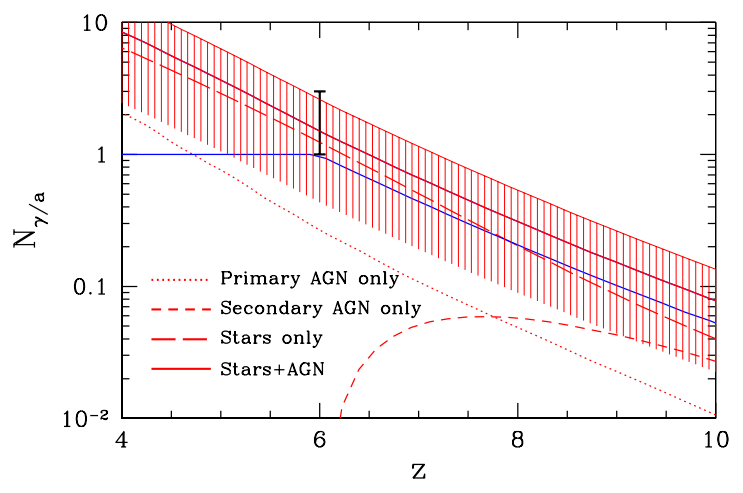

FIG. 5.- The total ionizations-to-atom ratio as a function of redshift our fiducial model ("large seeds, high spins" in WMAP-5UP cosmology - red lines). The hatched area shows our estimate of the observational uncertainty (for a given theoretical model). The blue line shows the effective ionization fraction $x_{\mathrm{eff}}$ from equation (7). Dotted, short-dashed, and long-dashed red lines show the contributions from primary and secondary ionization from quasars and from stars respectively.

sidered as an upper limit; we emphasize this by having the hatched area in Fig. 5 unclosed from below. We also show the evolution of the effective ionization fraction $x_{\text {eff }}$ as an illustrative (but highly approximate) reionization history.

\section{DISCUSSION}

Since our quasar models cover a wide range of physically plausible possibilities, we can draw some general conclusions from Fig. 3 and 4 In particular, we notice that (i) by $z \approx 6$ the quasar contribution to the total ionization budget becomes mostly sub-dominant, not exceeding 50\% in the best case, and likely falling below $20 \%$ or so. Never-the-less, at $z \approx 8$ in all our models quasars contribute from over $50 \%$ up to $90 \%$ of ionizations. This conclusion is particularly important to theoretical modeling and future observational measurements of the expected redshifted $21 \mathrm{~cm}$ emission from neutral hydrogen in the reionization era. While most of the currently planned pathfinder experiments rely, in part, on the existing simulations for critical design decisions, the predictions from those simulations - none of which include a quasar contribution - become inaccurate at $z \gtrsim 8$. It is, therefore, important to keep in mind that much theoretical work still needs to be done before even the basic observables for the incoming $21 \mathrm{~cm}$ experiments (like the fluctuation power spectrum) can be computed theoretically with $\sim 20 \%$ precision (within a given cosmological model).

Most of the contribution to the quasar ionizing budget is dominated by MBHs with mass $<10^{6} \mathrm{M}_{\odot}$. Such small, lowluminosity MBHs do not contribute to the bright end of the luminosity function of quasars, and are therefore difficult to account from simple extrapolations of the luminosity function of quasars. These small holes are not hosted in extremely massive galaxies residing in the highest density peaks (5 to $6 \sigma$ peaks), but are instead found in more common, "normal" systems, $\sim 3 \sigma$, peaks. Future generation of space-based telescopes, such as JWST and IXO, are likely to detect and constrain the evolution of the population of accreting massive black holes at early times $(z \lesssim 10)$.

We are grateful to the anonymous referee for constructive and ensightful comment and to Andrey Kravtsov for the permission to use his halo mass function code free of charge. This work was supported in part by the DOE, by the NSF grant AST-0507596, and by the Kavli Institute for Cosmological Physics at the University of Chicago (NG). Support for this work was also provided by NASA through Chandra Award Number TM9-0006X (MV).

\section{REFERENCES}

Begelman, M. C., Rossi, E. M., \& Armitage, P. J. 2007, ArXiv e-prints, 711 Begelman, M. C., Volonteri, M., \& Rees, M. J. 2006, MNRAS, 370, 289 Belikov, A. V. \& Hooper, D. 2009, ArXiv e-prints

Bouwens, R. J., Illingworth, G. D., Franx, M., \& Ford, H. 2007, ArXiv e-prints, 707

-. 2008, ApJ, 686, 230

Cohn, J. D. \& Chang, T.-C. 2007, MNRAS, 374, 72

Dijkstra, M., Haiman, Z., \& Loeb, A. 2004a, ApJ, 613, 646

-. 2004b, ApJ, 613, 646

Furlanetto, S. R. 2006, MNRAS, 371, 867

Giroux, M. L. \& Shapiro, P. R. 1996a, ApJS, 102, 191

-. 1996b, ApJS, 102, 191

Gnedin, N. Y. 2008, ApJ, 673, L1

Hopkins, P. F., Richards, G. T., \& Hernquist, L. 2007, ApJ, 654, 731

King, A. R. \& Pringle, J. E. 2007, MNRAS, 377, L25

Komatsu, E., Dunkley, J., Nolta, M. R., Bennett, C. L., Gold, B., Hinshaw,

G., Jarosik, N., Larson, D., Limon, M., Page, L., Spergel, D. N., Halpern,

M., Hill, R. S., Kogut, A., Meyer, S. S., Tucker, G. S., Weiland, J. L.,

Wollack, E., \& Wright, E. L. 2009, ApJS, 180, 330

Kramer, R. H. \& Haiman, Z. 2008, MNRAS, 385, 1561

Lodato, G. \& Natarajan, P. 2006, MNRAS, 371, 1813

Machacek, M. E., Bryan, G. L., \& Abel, T. 2003, MNRAS, 338, 273

Madau, P., Haardt, F., \& Rees, M. J. 1999, ApJ, 514, 648

Madau, P. \& Rees, M. J. 2001, ApJ, 551, L27

Madau, P., Rees, M. J., Volonteri, M., Haardt, F., \& Oh, S. P. 2004, ApJ, 604, 484

Merloni, A. \& Heinz, S. 2008, MNRAS, 388, 1011

Milosavljevic, M., Bromm, V., Couch, S. M., \& Oh, S. P. 2008, ArXiv e-prints
Oh, S. P. 2001, ApJ, 553, 499

Pelupessy, F. I., Di Matteo, T., \& Ciardi, B. 2007, ApJ, 665, 107

Ricotti, M., Gnedin, N. Y., \& Shull, J. M. 2002, ApJ, 575, 33

Ricotti, M. \& Ostriker, J. P. 2004, MNRAS, 352, 547

Ripamonti, E., Mapelli, M., \& Zaroubi, S. 2008, MNRAS, 387, 158

Salvaterra, R., Haardt, F., \& Ferrara, A. 2005, MNRAS, 362, L50

Salvaterra, R., Haardt, F., \& Volonteri, M. 2007, MNRAS, 374, 761

Santos, M. G., Amblard, A., Pritchard, J., Trac, H., Cen, R., \& Cooray, A. 2008, ApJ, 689, 1

Schleicher, D. R. G., Banerjee, R., \& Klessen, R. S. 2008, Phys. Rev. D, 78, 083005

Shapiro, P. R. \& Giroux, M. L. 1987, ApJ, 321, L107

Shapiro, P. R., Giroux, M. L., \& Babul, A. 1994, ApJ, 427, 25

Shapley, A. E., Steidel, C. C., Pettini, M., Adelberger, K. L., \& Erb, D. K.

2006, ApJ, 651, 688

Shull, J. M. \& van Steenberg, M. E. 1985, ApJ, 298, 268

Shull, J. M. \& Venkatesan, A. 2008, ApJ, 685, 1

Thomas, R. M. \& Zaroubi, S. 2008, MNRAS, 384, 1080

Valdés, M. \& Ferrara, A. 2008, MNRAS, 387, L8

Venkatesan, A., Giroux, M. L., \& Shull, J. M. 2001, ApJ, 563, 1

Volonteri, M., Haardt, F., \& Madau, P. 2003, ApJ, 582, 559

Volonteri, M., Madau, P., Quataert, E., \& Rees, M. J. 2005, ApJ, 620, 69

Warszawski, L., Geil, P. M., \& Wyithe, S. 2008, ArXiv e-prints

Zaroubi, S., Thomas, R. M., Sugiyama, N., \& Silk, J. 2007, MNRAS, 375,

1269 